\title{
Ion Clock Makes Maiden Space Voyage
}

\author{
After a year in low Earth orbit, a NASA demo mission proves that an \\ ultraprecise atomic clock retains its stability in space, paving the way for \\ real-time deep space navigation.
}

\section{By Rachel Berkowitz}

6 'Second star to the right and straight on 'til

morning" worked for Peter Pan, but in real life, Solar System travel is more complicated. Deep space navigation relies on measuring the transit time of beamed radio signals between a spaceship and Earth. Currently, all timing measurements are performed with Earth-based atomic clocks, which means a spaceship could wait up to several hours before its position information is relayed back to it. Autonomously performing those timing measurements onboard would greatly reduce the processing time. Eric Burt and his colleagues at NASA's Jet

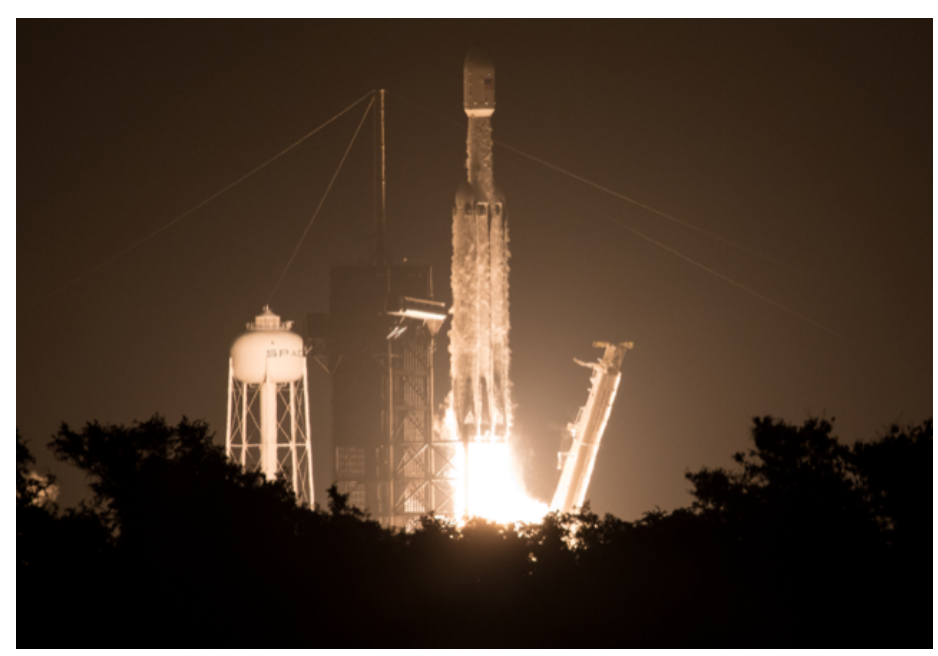

The Deep Space Atomic Clock launched aboard a SpaceX Falcon Heavy rocket in June 2019 and was powered on in August. Now it's returned nearly two years' worth of data, proving the capabilities of a trapped-ion clock to operate autonomously in space.

Credit: NASA/Joel Kowsky
Propulsion Laboratory in California now report the first successful test of a compact trapped-ion atomic clock in an orbiting spacecraft. The Deep Space Atomic Clock (DSAC) demonstrated long-term stability that exceeds current space clocks by an order of magnitude.

The most common clocks aboard space probes are designed to be small and have stable short-term performance, at the expense of long-term stability. That's in contrast to the atomic clocks used on Earth and on GPS satellites, where long-term stability can be important. Atomic clocks keep time by locking the frequency of an oscillator to the extremely stable frequencies associated with internal atomic states. GPS atomic clocks and conventional space clock designs rely on confining atoms in a box, where collisions with the box walls hamper performance and result in errors of about $1 \mathrm{~ns}$ after 1 day-a seemingly small amount, but one that could add up to navigational disaster on a years-long deep space voyage.

To optimize long-term stability, metrology researchers have developed trapped-ion atomic clocks which capture, cool, and excite ions using electromagnetic fields. "Several decades ago, it was realized that trapping ions could revolutionize atomic physics in general, and shortly after that, atomic clocks," Burt says. However, putting trapped-ion atomic clocks in space poses several challenges, starting with the violent conditions of the launch and extending to the extreme radiation environment of space.

Burt and his colleagues have met these challenges with the DSAC. At the heart of the clock's design is a small cloud of mercury ions trapped by electric fields. Microwave pulses 
generated by the clock are directed into the cloud, and some of the ions respond by changing their energy state. The number of ions that change state is related to how close the microwave pulse is to the right frequency. By measuring this number, a frequency error can be calculated and used to correct the frequency of a quartz oscillator that's integrated into the clock's ticking mechanism. This technology establishes the nearly perfect $40.5 \mathrm{GHz}$ "tick" of the clock. The design avoids lasers, cryogenics, or microwave cavities, thereby enabling a small and robust device that consumes less than $50 \mathrm{~W}$ of power. Whereas an Earth-based atomic clock takes the space of a refrigerator, the DSAC clock is the size of a toaster.

In laboratory tests, the researchers operated the DSAC technology continuously for nine months, observing a drift in the clock's stability that corresponded to a 1-ns error over 20 days. That drift measurement came from tracking how much the clock's frequency changed relative to the established value of $40.5 \mathrm{GHz}$. For example, a $4-\mathrm{GHz}$ change would correspond to $10 \%$ drift, or a deviation of 0.1 ; in DSAC's case, the drift was a mere $2.7 \times 10^{-17}$ deviation per day.

For their space test, the team placed the DSAC on the General Atomics' Orbital Test Bed spacecraft, which was launched into low Earth orbit in June 2019 by a SpaceX Falcon Heavy rocket. This space-faring clock, which used a slightly simpler trapping mechanism than used in the lab test, has now returned more than 12 months of data. Through comparisons with terrestrial clocks and GPS satellite signals, Burt and his JPL colleagues determined that the clock's frequency drifted by just $3 \times 10^{-16}$ per day. That frequency shift corresponds to a 1-ns error over eight days, exceeding the stability of other space clocks by an order of magnitude. "As far as performance goes, the long-term stability was a huge success," Burt says. "Another main success of the technology was its ability to operate continuously without interactions from the ground."

Nils Huntemann, a staff scientist at Germany's National Metrology Institute, points out that the mercury ion trap is the culmination of several decades' worth of work. "The big advantage is that the system is intrinsically drift-free," he says.
"The DSAC flight is the first step in bringing higher clock stability and accuracy to space, and there's still a lot of potential for advancement in the technology."

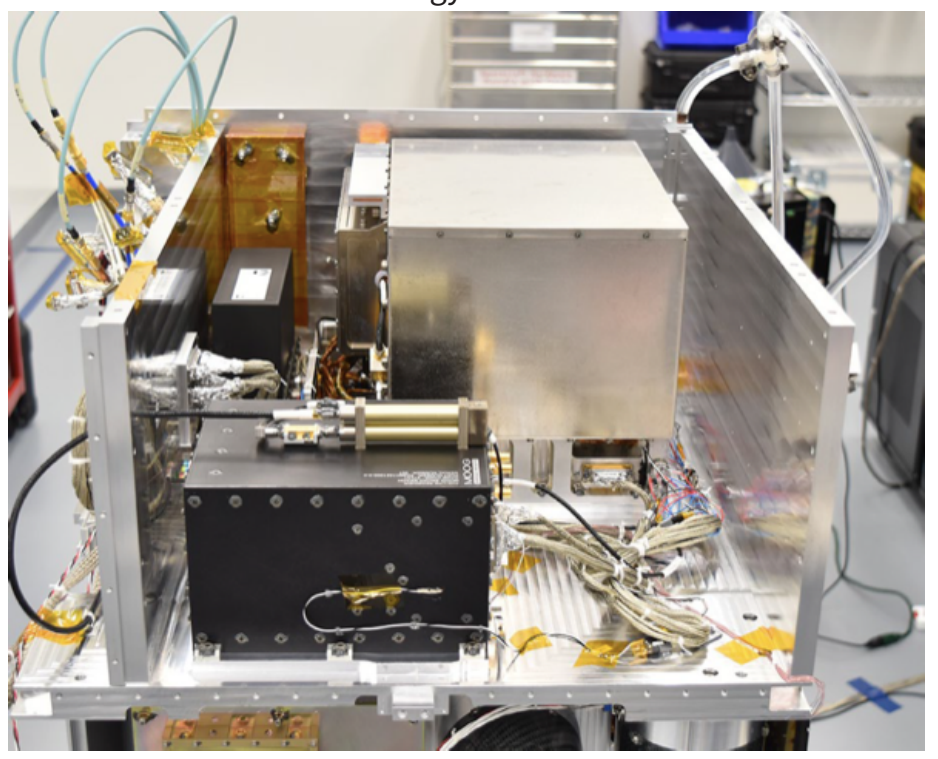

The Deep Space Atomic Clock instrument (silver box in the back) is shown here with other instruments that were included as part of the scientific payload on the General Atomics' Orbital Test Bed spacecraft.

Credit: General Atomics

The technology has demonstrated its suitability for applications that require autonomous operation. Future use could allow spacecraft to focus on mission objectives and on navigating in real time, rather than constantly orienting their antennae earthward to receive position information and maneuver instructions. Eventually, sending multiple units into orbit around other moons or planets could provide a GPS-like navigation system for ground-based rovers or even human explorers.

Rachel Berkowitz is a Corresponding Editor for Physics based in Vancouver, Canada. 